\title{
Achieving spatial coherence in German Sign Language narratives: The use of classifiers and perspective
}

\author{
Pamela M. Perniss \\ Max Planck Institute for Psycholinguistics, Postbus 310, 6500 Nijmegen, The Netherlands \\ Received 1 April 2004; received in revised form 29 March 2005; accepted 7 June 2005 \\ Available online 9 March 2006
}

\begin{abstract}
Spatial coherence in discourse relies on the use of devices that provide information about where referents are and where events take place. In signed language, two primary devices for achieving and maintaining spatial coherence are the use of classifier forms and signing perspective. This paper gives a unified account of the relationship between perspective and classifiers, and divides the range of possible correspondences between these two devices into prototypical and non-prototypical alignments. An analysis of German Sign Language narratives of complex events investigates the role of different classifier-perspective constructions in encoding spatial information about location, orientation, action and motion, as well as size and shape of referents. In particular, I show how non-prototypical alignments, including simultaneity of perspectives, contribute to the maintenance of spatial coherence, and provide functional explanations in terms of efficiency and informativeness constraints on discourse.
\end{abstract}

(C) 2006 Elsevier B.V. All rights reserved.

Keywords: Classifiers; Perspective; Spatial coherence; Efficiency constraint; Informativeness constraint; Simultaneity

\section{Introduction}

To follow a complex event in narrative, listeners must construct a mental representation of the spatial layout being described and understand the nature of the spatial relationships between referents. This means knowing where referents are, relative to other referents and integrating information about the movement and activities of these referents into a full representation of the event space. The success with which this is done relies to a large extent on the maintenance of coherence by the narrator. Maintaining coherence in a narrative means achieving consistency and

Abbreviations: CL, classifier form; RH, right hand; LH, left hand; biman, bimanual; opp, opposite

E-mail address: Pamela.Perniss@mpi.nl. 
continuity with respect to different types of information, including referent identification, and temporal, causal, locational, and, importantly, spatial structure (Gernsbacher, 1997; Givón, 1995).

Spatial coherence in narratives depends on the use of lexical or grammatical cues about where referents are and where events take place. The placement of cues allows the addressee to conceptually construct the spatial setting(s) in which an event or series of events take(s) place. In sign language, two key devices for cueing spatial information are the use of classifier predicates and the type of spatial representation. Topographic spatial representation in space is evident in two types of signing perspective. In one case, the event space is conceptualized as life-sized and the signer is within the event, in the role of a character. In the other case, the signer is external to the event, and the event space is reduced in size to the area of space in front of the signer's body and involves classifier predicates. In the use of classifier predicates, the handshape represents the whole referent or the handling of a referent, and the position of the hand in space encodes information about location, orientation, and/or motion.

The purpose of this paper is to show how signers of German Sign Language (Deutsche Gebärdensprache, DGS) use classifier forms and signing perspective in different ways in order to achieve spatial coherence in narratives. Previous accounts of the use of these cues implicitly assume certain correspondences between classifier forms and spatial representation that are motivated by recourse to argument structure and verb semantics. Classifiers representing the handling of objects (i.e. transitive event types) occur within a life-sized character perspective event space, while classifiers representing the location and motion of objects (i.e. intransitive event types) occur within a model-sized observer perspective event space. In this paper, these correspondences are called prototypical alignments of perspective and classifiers. In extended discourse, however, the range of encountered representation types is more varied, and not restricted to prototypical alignments. This paper is concerned especially with the occurrence and function of non-prototypical alignments of classifiers and perspectives in discourse. A detailed analysis of two pairs of German Sign Language narratives describes the use of both prototypical and non-prototypical alignment constructions in encoding information about spatial relationships. The function of the non-prototypical alignments is explained on the basis of efficiency of expression and informativeness constraints on discourse structure.

\section{Background}

\subsection{Signing perspectives}

With respect to the localization of referents in sign space, two different types of spatial representation or signing perspective can be identified. The size of the event space, the vantage point on the event, the forms used for referent representation, and the type of information that is expressed are the main diagnostic features that distinguish the two types. Notably, the place the signer occupies with respect to the represented event is an important diagnostic feature. In one type of spatial representation, the signer is conceptualized as being within the event space, effectively playing the part of a character on stage. In the role of a character, the signer "constructs" the actions and attitudes attributed to this character (Metzger, 1995). The event is conceptualized on a life-sized scale in the space around the signer's body. Signing that reflects this type of event space conceptualization is referred to as character perspective signing, in this paper. In the other type, called observer perspective here, the signer is outside of the event conceptually, viewing the scene from the perspective of an external observer. The signer is like a 
puppeteer, manipulating the characters that appear on stage and constructing the event on a reduced scale in the area of space in front of the body.

Researchers interested in the use of space in discourse or spatial description in sign language have given different name to the two types of spatial representation, e.g. viewer versus diagrammatic spatial format (Emmorey and Falgier, 1998), surrogate versus depictive space (Liddell, 2003), ${ }^{1}$ participant versus global viewpoint (Dudis, 2004), shifted versus fixed referential framework (Bellugi and Klima, 1990; Morgan, 1999), or protagonist versus narrator perspective (Slobin et al., 2003). Moreover, McNeill (1992) makes a similar distinction for gesture that accompanies speech in defining a character versus an observer viewpoint. For the purposes of this paper, the overlap between the criteria put forward to distinguish between the types of representation in each case is taken to be large enough to assume that these are different names for the same phenomena. The differences in terminology are thus not dealt with further here.

\subsection{Classifier predicates}

Classifier predicates are used to schematically represent the existence, location, and motion of referents in sign space and have been the focus of much research in sign linguistics. Their use has been described for the majority of sign languages studied to date (cf. Schembri, 2003 for a list of over 30 sign languages). ${ }^{2}$ In these predicates, the handshape reflects certain salient - usually geometric - properties of the referents being talked about. Different types of classifiers are identified depending on how referents are depicted. Four main types of classifier handshapes have been identified: (1) entity/whole object, where the hand represents a referent as a whole; (2) handling/instrument, where the hand represents the manipulation of a referent or depicts the referent used as an instrument; (3) limb/body part, where the hands represent the limbs of an animate referent; and (4) extension/contour, where the movement of the hands traces the contours of the referent (cf. Benedicto and Brentari, 2000; Engberg-Pedersen, 1993; McDonald, 1982; Schick, 1990; Supalla, 1986 for slightly different categorizations).

In DGS, for example, vehicles with four wheels are represented by the rectangular shape of the flat hand, fingers outstretched, palm facing down (B-hand). The handshape here represents the entire vehicle, and is thus a whole entity classifier. The handshape in handle classifiers depicts the manipulation or handling of a referent. Geometric features of referents are reflected in the handshape, since the hand imitates holding a particular type of object. Holding a glass, e.g. is represented by a $\mathrm{C}$-handshape, where the fingers and thumb curve around the imagined round shape of a glass. The handshapes used for limb classifiers differ depending on which type and which part of the limb is represented. Two fists (S-hands), knuckles oriented down, for example, can represent the large front feet of an elephant, while two extended index fingers (G-hands), pointing down, can represent a person's legs. Finally, entities can be depicted by specifying geometric form or extension. The shape of a fence around an enclosure, for example, can be traced using two hands with four fingers extended upward and spread apart (four-hands).

\footnotetext{
${ }^{1}$ In previous work (cf. Liddell, 1994, 1995), a distinction was made between surrogate and token space.

2 The use of the term "classifier" to characterize the linguistic function of these predicates is a contentious issue in sign linguistics (cf. Cogill-Koez, 2000; Engberg-Pedersen, 1993; Liddell, 2003; Schembri, 2003; Slobin et al., 2003). Nevertheless, the term has been widely adopted and will not be problematized here.
} 


\subsection{The relationship between signing perspective and classifier forms}

The relationship between classifier forms and signing perspective is not often directly addressed in the literature. The mapping of referents onto what corresponds to observer perspective space has been investigated with respect to the morphological structure of entity classifiers (cf. Boyes-Braem, 1990; Emmorey, 1996; Supalla, 1986, 2003; Sutton-Spence and Woll, 1999; Talmy, 2003). Descriptions of signing in character perspective as constructed action or role shift only marginally mention the explicit use of handling classifiers (cf. Metzger, 1995; Liddell and Metzger, 1998; Padden, 1990; Meier, 1990; Aarons and Morgan, 2003).

Where the relationship between the use of classifiers and the use of space for referent mapping is addressed, it is generally in terms of argument structure or verb semantics (Zwitserlood, 2003; Engberg-Pedersen, 1993; McDonald, 1982). In these analyses, there is a systematic correspondence between entity classifiers and intransitive verbs, on the one hand, and handling classifiers and transitive verbs, on the other hand. ${ }^{3}$ Thus, the expression of motion and location aligns with the use of entity classifiers, while the expression of object manipulation aligns with the use of handling classifiers. Although most argument structure analyses do not explicitly discuss signing perspective, the prototypical manifestations of character and observer perspective with respect to classifier alignment can be motivated by the relationships assumed in these analyses.

\section{Present study}

This paper looks at how signers of German Sign Language use perspective and classifier forms to achieve spatial coherence in narratives. A unified account of the relationship between perspectives and classifier forms is given, which identifies the possible correspondences between the two factors, including both prototypical and non-prototypical alignments. The occurrence of constructions with different alignments is functionally explained in terms of efficiency and informativeness constraints on discourse structure.

The paper is structured as follows. The rest of this section elaborates and motivates the relevant concepts of perspective and classifier alignment (sections 3.1 and 3.2) in relationship to information type (section 3.3), and organization in discourse structure (section 3.4). After introducing the data (section 4), discourse analyses of signed DGS narratives are presented in sections 5 and 6 . The effect of the efficiency constraint on the possible constructions used to create a conceptual mapping between meaningful locations in observer and character perspective space is the focus of section 5. Section 6 discusses the use of prototypical and non-prototypical classifier-perspective alignments to represent information about relative scale. Finally, a summary and discussion are found in section 7.

\subsection{Perspective and classifier forms: prototypical alignment}

As introduced in section 2.3, the prototypical alignment between classifier forms and perspective is generally encountered in the literature as an implicit assumption of argument structure analyses. The handshape encodes the subject argument in intransitive verbs that express motion and location, and encodes the object argument in transitive verbs that express the transfer or

\footnotetext{
${ }^{3}$ McNeill (1992:119) makes a similar claim with respect to the distribution of occurrences of observer and character viewpoint gestures, i.e. that character viewpoint gestures appear more with transitive verbs, while observer viewpoint gestures appear more with intransitive verbs.
} 
manipulation of objects (Zwitserlood, 2003). However, the prototypical manifestations of character and observer perspective in signing are not fully captured by recourse to argument structure. Two further factors are presented here as influencing the manifestation of signing in the two perspectives, as determined by the representation of the locations, action, and motions of referents.

Firstly, articulatory constraints inherent in certain forms of signing play a role in determining the representation type. For example, to depict manual activity of a character, the animate referent is mapped onto the signer's body and a handling classifier that identifies the referent held in the hand is used. This aligns with character perspective signing. However, showing change of location, i.e. as path movement, is problematic in character perspective, because it would require the signer to actually move her own body. Thus, path movement must be represented via entity classifiers by the movement and location of the signer's hands in space. The use of entity classifiers and the representation of change of location aligns with observer perspective. Thus, typically, motion and location are expressed within an event space projection that corresponds to observer perspective, while object manipulation is expressed in a life-sized event space that corresponds to character perspective.

Secondly, the conceptualization of the spatial setting in which a complex event takes place determines the manifestation of observer or character perspective signing, in that it has an influence on the placement of referents in space. In extended discourse, the representation of referent location, action, and motion depends on how event space is conceptually projected onto sign space. The representation of interaction between two animate referents, for example, differs depending on the way in which event space is conceptualized. In the conceptually life-sized event space in character perspective signing, a second animate referent, with whom the animate referent mapped onto the signer's body interacts, is canonically located opposite the signer. Thus, motion is directed along the sagittal axis. In observer perspective signing, however, where the signer is not a part of the event, the two animate referents are canonically located opposite each other to the left and right of the signer's body. Here, the motion is directed along the lateral axis.

In sum, the prototypical manifestations of the two signing perspectives can be characterized in terms of the size of the event space, the type of classifiers that occur, and the main direction of movement of the sign depicting motion. These elements are summarized in Fig. 1.

\begin{tabular}{|c|c|c|}
\hline & Character perspective & Observer perspective \\
\hline $\begin{array}{l}\text { Event space } \\
\text { projection }\end{array}$ & $\begin{array}{l}\text { - } \quad \text { Life-sized (space } \\
\text { surrounding signer) } \\
\text { - } \quad \text { Signer part of event }\end{array}$ & $\begin{array}{l}\text { - Model-sized (space in } \\
\text { front of signer) } \\
\text { - Signer outside of event }\end{array}$ \\
\hline Classifiers & $\begin{array}{l}\text { - Handling } \\
\text { - Large-scale SASS }\end{array}$ & $\begin{array}{ll}\text { - } & \text { Entity } \\
\text { - Small-scale SASS }\end{array}$ \\
\hline Direction & - Sagittal axis & - Lateral axis \\
\hline
\end{tabular}

Fig. 1. Prototypical manifestations of signing perspective.

\subsection{Perspective and classifier forms: non-prototypical alignment}

The prototypical alignments of event space projection and classifier forms shown in Fig. 1 do not reflect the much greater range of construction types actually encountered in signing, i.e. in extended discourse. The elements that contribute to the topographic structuring of space (i.e. the 
size of the projected event space, the use of classifiers, and the direction of the moving sign) can be differentially combined. For example, a life-sized conceptualization of event space, as in character perspective signing, does not preclude the use of entity classifiers. The relationship between perspective and classifier forms is thus more complex than argument structure analyses or morphosyntactic constraints alone account for. A unified account of the occurrence of both prototypical and non-prototypical alignments of perspective and classifiers is needed in order to grasp the complexity of the relationship and to be able to give functional explanations of the forms encountered in discourse.

In the literature, non-prototypical alignments have not been widely discussed. Liddell (2000) uses mental spaces and conceptual blending theories to explain the mapping of elements from conceptual event space onto different articulators and/or locations in space. The existence of elements corresponding to more than one perspective in a single representation are straightforwardly explained under the conceptual blending approach (cf. also Liddell, 1998, 2003). Two different perspectives on an event - a "zoomed in" and a "zoomed out" view - can be simultaneously represented in what he calls a "simultaneous blend." For example, a signer can depict a car stopped at an intersection (i.e. a "zoomed out" view of the event) by mapping the car onto his left hand with an entity classifier, and at the same time can depict the driver of the car looking both ways before proceeding to drive (i.e. a "zoomed in" view of the event) by mapping the driver onto his body and turning his head side to side. Similarly, Dudis (2004) uses a conceptual blending framework to describe the possibility of simultaneous perspectives through the involvement of different "partitionable zones" of the body. Multiple elements present in the conceptual event space can be made simultaneously visible in the real space by mapping them onto different parts of the body. Aarons and Morgan (2003) examine the creation of what they call multiple perspectives by describing the use of entity and handle classifiers in episodes of constructed action. They present similar examples of a simultaneous "zoomed in" and "zoomed out" view of an event.

The range of possible correspondences between classifier forms and signing perspective are represented in Fig. 2. Classifiers and event space are treated here as independent factors that can appear in various combinations. It will be argued in the rest of the paper that the different possible combinations can fulfill different functions with respect to discourse coherence. The focus of investigation here is specifically on the function of non-prototypical alignments.

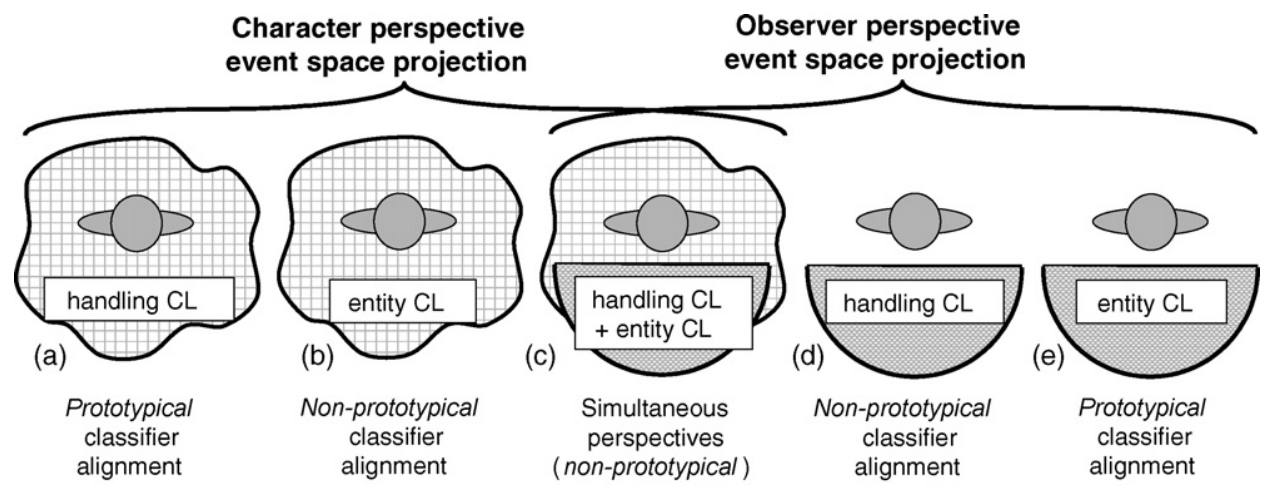

character perspective

observer perspective

Fig. 2. Possible correspondences between signing perspective and classifier forms. 
In the figure, the wavy line surrounding the signer (bird's eye view of head and shoulders) in Fig. 2(a-c) indicates a character perspective event space projection (cf. Fridman-Mintz and Liddell, 1998 for the use of this symbolic depiction). The semi-circle in front of the signer in Fig. 2(c-e) represents an observer perspective event space projection. Prototypical signing in character perspective is depicted by the correspondence in Fig. 2(a). Here, handle classifiers and SASS forms depict referents as life-sized. Prototypical signing in observer perspective is depicted by the correspondence in Fig. 2(e). In observer perspective event space, the size of referent representation using entity classifiers and SASSs is correspondingly smaller in size.

By treating classifiers and event space projection as independent factors, their co-occurrence is not restricted to these prototypical alignments. That is, the occurrence of the three types of nonprototypical correspondences, Fig. 2(b-d), is also accounted for. Specifically, the occurrence of entity classifiers and small-scale size and shape specifiers is not restricted to an observer perspective event space (Fig. 2(b)). Likewise, handling classifiers and large-scale size and shape specifiers do not occur only in a life-sized event space, i.e. in character perspective signing (Fig. 2(d)). ${ }^{4}$ Moreover, the prototypical alignments can be simultaneously combined (Fig. 2(c)). Through the simultaneous use of forms (prototypically) associated with each scale on different articulators, both event spaces are simultaneously active (cf. Perniss, 2003, in press for further descriptions of the occurrence and function of such simultaneous perspective constructions).

Taken together, the constructions displaying non-prototypical alignments appear frequently in signed narratives in German Sign Language. Taken separately, there are significant differences in their relative frequency of occurrence. Entity classifiers in a character perspective event space occur as the most prevalent mixed type in DGS (Fig. 2(b)). In comparison, occurrences of handle classifiers in observer perspective (Fig. 2(d)) and simultaneous perspective constructions (Fig. 2(c)) are quite rare. There is evidence, however, that the frequency distribution may differ cross-linguistically between sign languages. For example, preliminary investigation has revealed that Turkish signers make greater use of handle classifiers on an observer perspective scale, but much less use of character perspective with entity classifiers than German signers do (Perniss and Özyürek, accepted).

\subsection{Signing perspective and information type}

In this section, I will argue that the use of spatial devices in encoding action and interaction of animate and inanimate referents is dependent on information types expressed in the event. In particular, the focus is on how animate and inanimate referents get represented in transitive and intransitive event types. Transitive event types denote the manipulation of inanimate objects by animate referents, while intransitives denote the motion and location of animate and inanimate referents.

The expression of different information types corresponds to different types of representation. Fig. 1 in section 3.1 characterizes the prototypical manifestations of spatial representation types, i.e. of observer and character perspective, in terms of three distinguishing factors: (1) the size of the projected event space, (2) the type of classifiers that occur, and (3) the main direction of movement of the sign depicting motion. Of these factors, only the choice of classifier form is

\footnotetext{
${ }^{4}$ The space that determines the signing scale, as indicated by the wavy line around the signer (character scale) and the semi-circle in front of the signer (observer scale), entails the occurrence of the prototypically associated classifier forms in each case. Thus, both prototypically and non-prototypically associated forms occur in the non-prototypical classifier alignment constructions.
} 
truly restricted by the information type, as a result of articulatory constraints. Entity classifiers are needed to encode location and motion of referents, because referents must be mapped on the hands in order to depict movement across space. However, handling classifiers are necessary to encode manual activity and object manipulation, whereby animate referents get mapped onto the body. In contrast, it is not necessary that the event space projection and the direction of the moving sign be prototypically aligned with the classifier form. That is, both transitive and intransitive verb semantics can be represented either on the lateral or sagittal axis and either within an observer or character perspective event space.

Thus, the restrictions posed by the type of information in the event do not demand the use of fully prototypically aligned signing perspectives. In other words, the use of prototypical alignments in signing can be motivated by information type only with respect to the use of classifiers. The projection of event space and directionality can be either prototypically or nonprototypically aligned with the classifier forms that are needed to encode transitive events (i.e. handling classifiers) and intransitive events (i.e. entity classifiers), respectively.

In extended narrative discourse in DGS, non-prototypical alignments frequently appear. Information type may determine the use of classifier forms, but has no necessary influence on the other factors that contribute to the full manifestation of signing perspective. Thus, to explain the form and function of occurrences of non-prototypical alignments of perspective and classifiers in discourse, it is necessary to look not only at information type, but at overarching discourse structuring principles. The next section presents the choice of expressive elements in the light of efficiency and informativeness constraints in discourse.

\subsection{Signing perspective and discourse structure}

The explication of efficiency and informativeness principles in discourse date back to Grice's (1975) conversational maxims. Grice's two principles of quantity are formulated as "make your contribution as informative as required" (Q1) and "do not make your contribution more informative than is required" (Q2). In later research on pragmatic theory, the essence of these maxims were reformulated as the Q-principle (Principle of Quantity) and the I-principle (Principle of Informativeness), respectively (Horn, 1984; Atlas and Levinson, 1981; Levinson, 2000). ${ }^{5}$ In a discourse context, the two principles pull in opposite directions, minimizing the speaker's effort on the one hand (i.e. maximizing the speaker's efficiency via the I-principle), and minimizing the addressee's effort on the other hand (i.e. maximizing the hearer's input via the $Q$-principle).

In this paper, the principles of efficiency and informativeness serve as the basis for explanation of the occurrence of non-prototypical alignments of event space projection and classifier forms in discourse. The implicit assumption of a strict pairing of classifiers and event space, as is often encountered in the literature, does not hold up when one looks at actual discourse. Narrative discourse frequently displays the use of elements non-prototypically aligned with the event space projection. The analysis of the DGS narratives below presents the occurrence of non-prototypical alignments as the result of efficiency and informativeness constraints on discourse structure. The effect of these constraints is based on an assessment of spatial coherence, i.e. coherence with respect to spatial relationships in the event, achieved through the use of constructions with nonprototypical alignments of perspective and classifier forms.

\footnotetext{
${ }^{5}$ Horn uses the label R-principle for what Levinson calls the I-principle.
} 
I will show that, with respect to the expression of spatial relationships, the use of nonprototypically aligned constructions can serve spatial coherence both in terms of efficiency and informativeness. In one case, the signer's efficiency in unambiguously conveying locative information is increased. In the other case, there is an increase in informativeness about the exact nature of the spatial relationships between figure and ground objects. In short, the purpose of this paper is to show that the occurrence of different combinations of classifier forms and perspective, as shown in Fig. 2 (section 3.2), can be explained by efficiency and informative constraints and that their use plays an important role in the achievement and maintenance of spatial coherence in narratives.

\section{The data}

German Sign Language narratives were elicited from four different signers on the basis of two short cartoon stimulus clips featuring animate referents engaged in activities and interactions in a fixed event space. Pairs of signers were videotaped during data collection sessions. One signer watched the stimulus film and narrated the story to the second signer (telling), who then retold the story (retelling) without having seen the video clip. Thus, a telling and retelling counterpart are analyzed for both stimulus films. The narratives chosen for analysis in this paper are taken from a larger corpus of DGS data. ${ }^{6}$ Data were transcribed and coded using ELAN. ${ }^{7}$ Coding was for classifier forms, signing perspective, simultaneous constructions, locative constructions, and consistency in location-referent associations.

Two stimulus films were chosen from the corpus for analysis here. ${ }^{8}$ The analysis of the first stimulus film (section 5) focuses on the use of classifier forms to create a spatial mapping between locations that are assigned meaning in observer and character perspective event spaces. The narratives of this event illustrate a sequential (section 5.1) and a simultaneous discourse-structuring strategy (section 5.2), respectively, to achieve spatial coherence. The simultaneous strategy employs a non-prototypically aligned classifier-perspective construction, and is shown to be more efficient than the sequential strategy with respect to the expression of locative information. In the narrative of the second stimulus film (section 6), the focus of analysis is on the differential use of classifier forms to correspond to the scale of referent representation. One narrative exhibits prototypical alignments between classifier and event scale (section 6.1); the other, non-prototypical alignments (section 6.2). The narrative that employs non-prototypical alignments is more informative with respect to the exact nature of the depicted figure-ground relationships.

\section{Efficiency constraint: sequentiality versus simultaneity for mapping between perspectives}

In order to achieve spatial coherence in signed narratives that use both observer and character perspective representations, the correspondence between the two representations with respect to spatial locations has to be clear. The default convention for mapping between observer and

\footnotetext{
${ }^{6}$ The corpus consists of video recordings of elicited event narratives, spatial descriptions of object configuration, and route descriptions. In total, narratives were elicited from seven pairs of different signers for 18 cartoon stimulus clips, comprising five hours of recorded material. Recordings of natural conversation supplement the elicited material.

${ }^{7}$ ELAN is the European Distributed Corpora Linguistic ANnotator (ELAN), developed by members of the technical group at the Max Planck Institute for Psycholinguistics. It is similar in layout to SignStream (Neidle, 2002).

${ }^{8}$ See Appendices A and B for content descriptions of the two stimulus films used.
} 
character perspective event space projections proceeds from the assumption of prototypical alignments between classifiers and perspective and is determined by a linear sequence of prototypically aligned representations. Location and orientation information, represented in observer perspective with entity classifier forms, is followed by constructed action representations using the hands, face, and body in character perspective. In the character perspective representation, the depicted location and orientation information remains valid. A change in location and orientation must be indicated by a return to entity classifier usage in observer perspective. Achieving mapping in this way structures discourse sequentially with respect to the use of perspectives.

The sequential strategy is illustrated in boxes (1) and (2) in Fig. 3 below, which gives a schematic representation of the signed narrative analyzed in section 5. Both boxes (1) and (2) depict prototypically-aligned observer perspective signing, followed by prototypically-aligned character perspective signing. In box (1), locative information is represented using an entity classifier in observer perspective. The location and orientation of a referent is depicted in a certain location in sign space (identified with a dot and labelled $l o c_{1}$ in the figure). In the subsequent character perspective representation, depicting manual activity, the referent is understood to be located at $l o c_{1}$ (as indicated in the figure by the dot placed within the area depicting the signer's head). In what is schematized in box (2) in the figure, the signer again uses a prototypically-aligned observer perspective representation to depict the location/orientation change of the referent (as indicated by the dot labelled $l o c_{2}$ ). The representation of the referent's activity in $l o c_{2}$ follows in a prototypically-aligned character perspective representation. Thus, in mapping between perspectives through sequentiality, the representation of information about referents' changes of location and orientation vs. the representation of information about their manual activity is neatly divided between observer and character perspective, respectively. In



observer perspective event space

character perspective event space

Loc $_{1}$ : referent location/orientation \#1

$\mathrm{Loc}_{2}$ : referent location/orientation \#2

Fig. 3. Schematized use of sequentiaity and simultaneity of perspectives in discourse. 
each case, signing exemplifies the prototypical alignment between classifier form and spatial representation type.

A different strategy for mapping between perspectives makes use of a unique type of simultaneity afforded by the modality. In signing, it is possible to simultaneously represent referents on both hands, i.e. to use simultaneous constructions, to represent events occurring simultaneously or to keep backgrounded information visible while signing foregrounded information (Miller, 1994; Engberg-Pedersen, 1994) However, it is also possible to use simultaneous constructions to simultaneously represent the same event from different perspectives. The use of such a simultaneous perspective construction is described in section 5.2 (cf. Fig. 2 in section 3.2). Its occurrence in the signed narrative depicted in Fig. 3 above is illustrated in box (3).

In the simultaneous perspective construction, the signer represents spatial information associated to observer and character perspectives at the same time. Specifically, the signer indexes $l o c_{1}$ and $l o c_{2}$ in an observer perspective event space, while simultaneously indexing two orientations (represented as orientations of his torso) in a character perspective event space. By simultaneously representing both perspectives, the signer is able to distinctively link locations in space associated with observer perspective to locations associated with character perspective. To put it another way, the two orientations of the entity classifier in observer perspective are mapped to two distinct body orientations in character perspective. Once created, these mappings remain intact, and the signer continues his narrative by indicating changes from $l o c_{1}$ to $l o c_{2}$ in character perspective alone, implicitly invoking the corresponding observer perspective representations. This is illustrated in boxes (4) and (5) in Fig. 3.

\subsection{Mapping through sequentiality of perspectives in prototypical alignment}

The spatial relationship that is relevant to the analysis presented here is the orientation of the main protagonist (the mouse) with respect to the ground object (the stove). The mouse appears in two distinct orientations to the stove, either facing the stove (see still 1 in Appendix A), or turned $90^{\circ}$ to the left of the stove (see still 2 in Appendix A). In the course of the event, the mouse moves repeatedly back and forth between the two orientations. In both orientations, the mouse is handling an object (i.e. holding a frying pan with a pancake in it), and thus engaged in manual activity (see still 3 in Appendix A).

The signer begins his narrative by using entity classifiers to depict the spatial relationship between the mouse (inverted V-hand, 2-legged entity) and the stove (horizontal B-hand) (Fig. 4).

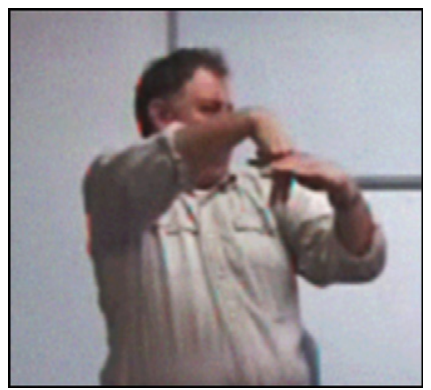

Fig. 4. Mouse(RH: entity CL)-stand-facing-stove(LH: entityCL). 


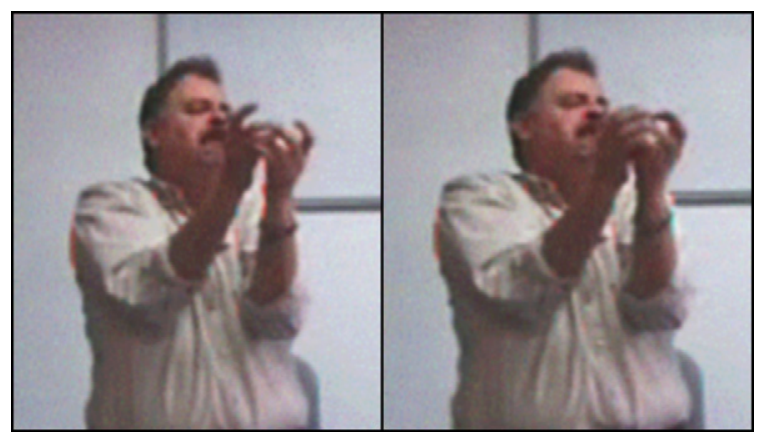

Fig. 5. Mouse(signer)-engage-in-unspecified-manual-activity.

This corresponds to the observer perspective part of box (1) in Fig. 3, where $l o c_{1}$ is depicted with prototypically-aligned entity classifiers.

To represent the mouse's manual activity in this orientation, the signer switches to a character perspective representation, mapping the mouse's body onto his own. The torso, shoulders, arms, hands, and face are all involved in depicting the mouse's activity (Fig. 5). ${ }^{9}$ Conceptually, here, the referents are located in a life-sized environment. The signer is the mouse (at location/ orientation $_{1}$ ) and the stove is located in the area in front of the signer. The character perspective utterance corresponds to the second part of box (1) in Fig. 3. The utterances in Figs. 4 and 5 both exemplify prototypical alignments of classifier form and event space, in each case expressing the type of information prototypically associated with each perspective.

To redefine the spatial relationship between the figure and ground objects, i.e. to depict the change of orientation of the mouse with respect to the stove, the signer again uses a two-legged entity classifier on an observer perspective scale (Fig. 6). This corresponds to the observer perspective part of box (2) in Fig. 3. The signer is external to the event space and depicts spatial information (i.e. the change from $l o c_{1}$ to $l o c_{2}$ ) using an entity classifier. Character perspective signing follows this, as indicated by the second part of box (2) in the schematization. Here, as is typical for character perspective, the signer is again within the event space, and is understood to be at location/orientation 2 (with the stove conceptually located to the right of the signer's body) (Fig. 7).

Structured in this way, the discourse is easy to understand and highly informative with respect to the expression of spatial relationships. The use of entity classifiers in observer perspective event space allows the change between two different orientations of the mouse to be clearly indicated, making the encoding of spatial information very exact. However, in terms of economy of expression, this discourse structure is not especially efficient. The use of only prototypicallyaligned combinations of perspective and classifiers means that the signer must continually switch between observer and character event spaces for the expression of change of location and orientation information and for the expression of manual activity and affective display, respectively. ${ }^{10}$

\footnotetext{
${ }^{9}$ The exact nature of the manual activity remains unspecified in the character perspective depiction, as the mouse is seen only from the back in this orientation in the stimulus film.

${ }^{10}$ Though no frames are shown here, the signer who retells this event makes exclusive use of the sequential structuring mechanism, relying solely on prototypical classifier-perspective alignments throughout her narrative.
} 


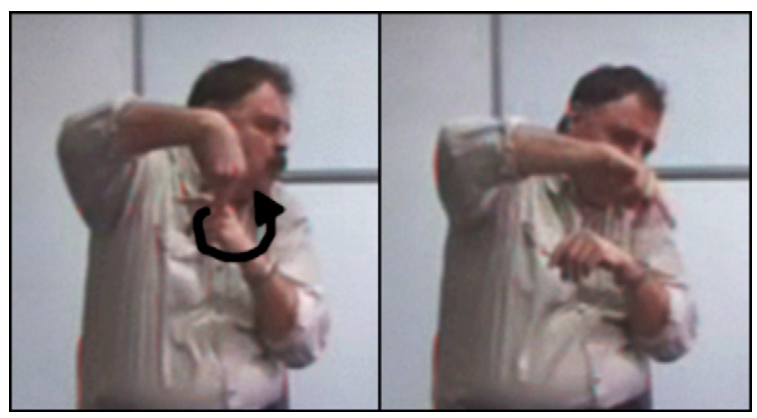

Fig. 6. Mouse(RH: entityCL)-turn-left(away from stove).

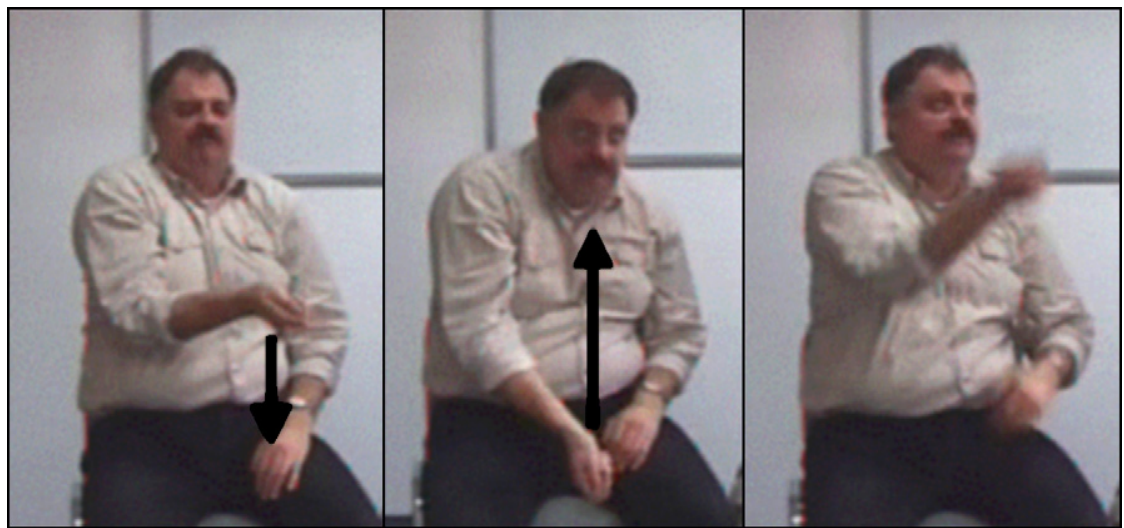

Fig. 7. Mouse(signer)-hold/flip-pan(RH: handlingCL).

In the next section, the use of a non-prototypically-aligned construction that makes unique use of the possibility of simultaneity is presented as a discourse-structuring mechanism. The construction simultaneously associates the two orientations of the mouse with locations in both observer and character event spaces, thereby creating a mapping between the two perspectives. Subsequently, the narrative remains maximally informative with respect to the encoding of spatial information, yet shows a substantial increase in efficiency or economy of expression.

\subsection{Mapping through simultaneity of perspectives in non-prototypical alignment}

The analysis presented here is of the same narrative as in the previous section. As shown, the signer structures the beginning of his narrative according to the sequential strategy. After establishing both location/orientation ${ }_{1}$ and location/orientation ${ }_{2}$ in observer perspective and subsequently, in each case, depicting the mouse's manual activity in character perspective, the signer employs a simultaneous perspective construction to map the referent orientations established in observer event space onto character event space (as shown in box (3) in Fig. 3).

In the signed narrative, the simultaneous perspective construction immediately follows the character perspective representation of the mouse's activity in $l o c_{2}$ (Fig. 7). The construction 


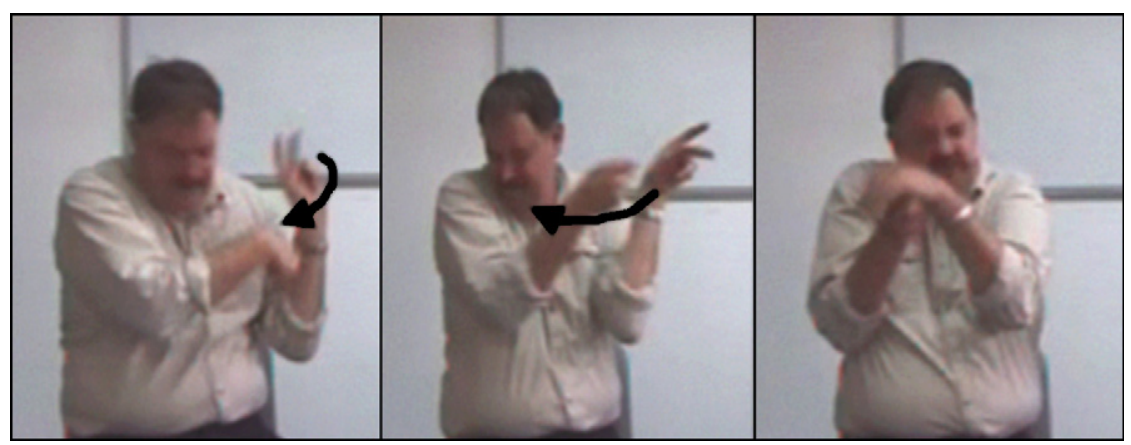

Fig. 8. Mouse(signer)-hold-pan(RH: handlingCL)-turn-right(to stove) + BACK/RETURN-TO(LH:loc 2 -to-loc 1 )

itself is manifested as a lateral turn of the hands, shoulders, and torso from left to right. The beginning location of the form indexes the mouse in location/orientation ${ }_{2}$, while the end location indexes location/orientation ${ }_{1}$ (Fig. 8). The life-sized environment that defines the event space projection of character perspective signing remains active through the continued presence of a handling classifier on the dominant (right) hand. Simultaneously, the spatial orientations specified in the observer perspective event space are accessed by a spatially modifiable lexical predicate meaning BACK/RETURN-TO, executed by the non-dominant (left) hand. ${ }^{11}$

Through the simultaneous construction, the beginning location of the dominant hand (holding the pan) and the corresponding orientation of the torso/shoulders (slightly to the left) is distinctively linked to the orientation of the mouse turned $90^{\circ}$ from the stove. In the same way, the end location of the dominant hand (holding the pan) and the corresponding orientation of the torso/shoulders (slightly to the right) is distinctively linked to the orientation of the mouse facing the stove. After the use of this non-prototypically aligned form, the signer can move between the two orientations of the body in character perspective without specifying the change in orientation in observer perspective (with an entity classifier) each time, as the sequential strategy would require. Instead, as depicted by boxes (4) and (5) in Fig. 3, the signer can stay in character perspective, shifting his body between two orientations that have become unequivocally associated with the two orientations of the mouse with respect to the stove (Figs. 9 and 10).

The signer's efficiency of information in the discourse is increased through the use of the simultaneous perspective construction. For equally precise encoding of spatial relationships, the signer who employs the simultaneous strategy needs only about half the number of utterances as the signer who used the sequential strategy throughout her narrative. The efficiency constraint on discourse structure motivates the use of the non-prototypically aligned form.

\footnotetext{
11 The sign executed by the non-dominant hand (BACK/RETURN-TO) is not an observer perspective form (i.e. an entity classifier), as they have been defined here. However, semantically, the spatial modification of the sign is understood with conceptual recourse to the spatial information previously specified in observer perspective using entity classifier forms. Technically, BACK/RETURN-TO can be analyzed as a sign produced in narrator perspective (by the signer as narrator) within an event space projected from observer perspective. The use of narrator perspective appears again in section 6.1 , but is otherwise not discussed, as it is not the focus of this paper (but cf. Perniss and Özyürek, submitted for publication, for an analysis that includes the use of narrator perspective).
} 


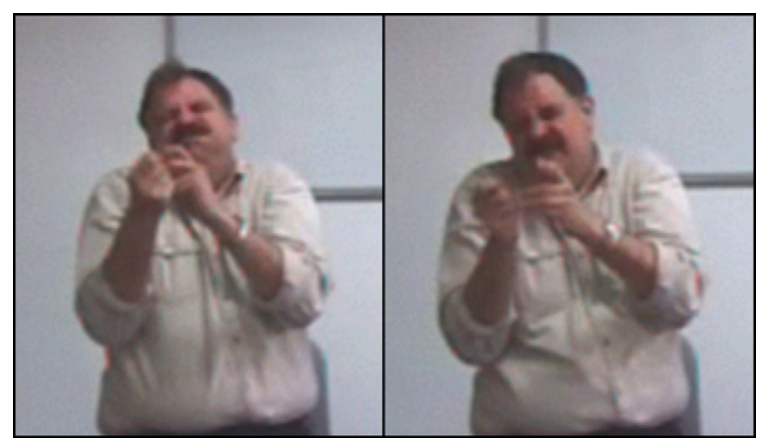

Fig. 9. Mouse(signer)-engage-in-unspecified-manual-activity.

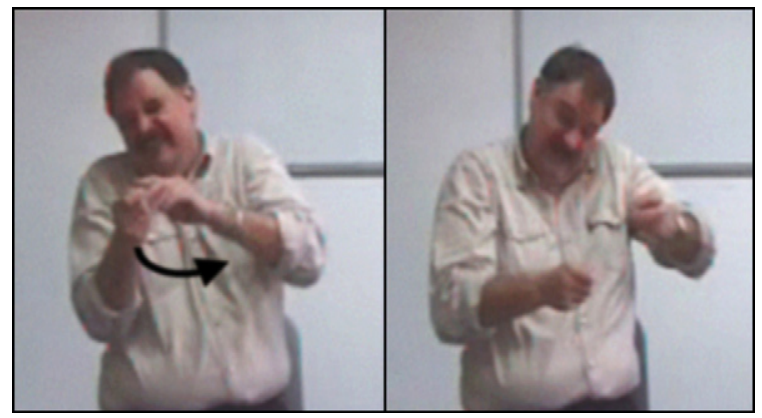

Fig. 10. Mouse(signer)-hold-pan(RH: handlingCL)-turn-left(away from stove).

The next section (section 6) presents the analysis of a telling and retelling narrative of the second stimulus film. In both narratives, the signer remains in character perspective, rendering the event in the role of an animate protagonist in a life-sized event space. Given this, the implicit assumptions about the co-occurrence of classifiers and perspective would predict that objects in the event that are handled by the animate mapped onto the singer's body be represented as life-sized.

In the prototypical manifestation of character perspective signing, the signer constructs actions (including the use handling classifiers if objects are manipulated), dialogue, thoughts, and attitudes of animate referents. Signers are not, however, restricted to "true" constructed action, even if they commit themselves to representation within a character event space projection throughout a narrative. As the comparison between sections 6.1 and 6.2 shows, both prototypical and non-prototypical alignments of classifiers and perspective are possible.

Moreover, the comparison shows that the informativeness of the narrative with respect to the encoding of the spatial relationship between a figure and ground object can be expressed differently by prototypical vs. non-prototypical alignments.

\section{Informativeness constraint: relative scale in referent representation}

In sign language, referents are visually represented in sign space based on salient physical features. This means that the representation of spatial relationships includes the specification of 
information about location, orientation, shape, as well as relative size or scale of the objects. As Slobin et al. (2003:19) note:

When using a polycomponential sign which depicts a figure moving or located with respect to a ground, it is critical that the scale of the figure and ground "classifiers" correspond with each other...(T)he same referent can be represented by different handshapes, depending on how their relative sizes are conceptualized in the projection from mental space into signing space.

Thus, in the representation of two objects - figure and ground - the relative scale of referent representation is important. In the narratives analyzed here, both signers choose to stay in character perspective throughout the narrative. In character perspective, where the event representation is on a life-sized scale, the assumption is that the representation of all referents should correspond in scale to the life-sized event space. This reflects the prototypical alignment of character perspective and handling classifiers, or constructed action signing. Some referents, however, cannot be easily represented on a life-sized scale. This means that, depending on the referents involved, it may not be possible to specify the exact nature of the spatial relationship between figure and ground with only life-sized referent representations. As is shown in the next two sections, the scale of referent representation affects the level of informativeness with respect to the encoding of figure-ground relationships.

In the stimulus film that forms the basis of analysis in this section, two animate referents (a mouse and an elephant) handle an inanimate referent (a ball) by passing it back and forth between them using different body parts (see Appendix B for the stimulus description and accompanying stills). The relevant spatial relationship is the relative size/scale of the ball, as figure object, compared to the different body parts, as ground objects, that the animate referents use to hit the ball (i.e. hands, feet, nose, and trunk). In the first narrative (section 6.1), the signer represents the event using only forms that correspond to the prototypical manifestation of character perspective signing. Representing the activity of kicking by actually using the feet is not felicitous in character perspective signing, however. Thus, the exact nature of the spatial relationship between the ball and the animate referent's feet (i.e. the figure contacting the ground) cannot be easily represented in prototypical character perspective signing. In contrast, the signer of the second narrative (section 6.2) uses non-prototypically-aligned classifier forms in character event space. Specifically, he uses classifier handshapes for the ball that correspond in scale to limb classifiers for the body parts used to hit the ball. The ball is not represented as life-sized, but rather with forms that correspond in size to observer perspective. As is shown below, his representation of the relative scale of referents with non-prototypically aligned forms achieves a higher degree of visual informativeness about the spatial relationships between figure and ground.

\subsection{Depiction of scale in prototypical alignment}

Prototypical character perspective signing - as "true" constructed action - means that all movements of the signer's hands, head, and body are attributable to the animate referent whose role the signer assumes. This means that body parts not isomorphic with or not felicitously represented with the signer's body cannot be depicted with other classifier forms. The adherence to prototypical, constructed action signing thus has certain consequences for the possible representation of figure and ground relationships in the narrative. In particular, in this event, several different body parts are used by the mouse and the elephant to hit the ball, i.e. the mouse's hands, snout, and feet, and the elephant's trunk. Of these, only the hands fulfill both conditions of 


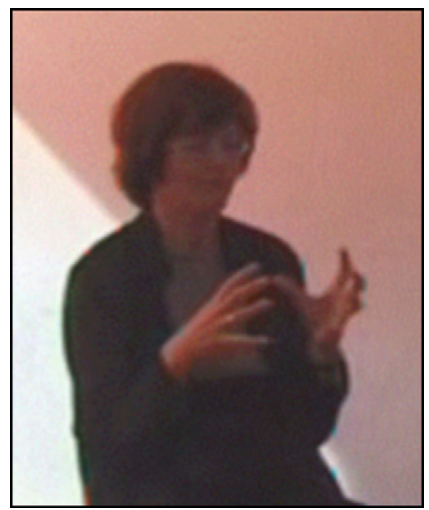

Fig. 11. Mouse(signer)-hold-ball(biman: handlingCL).

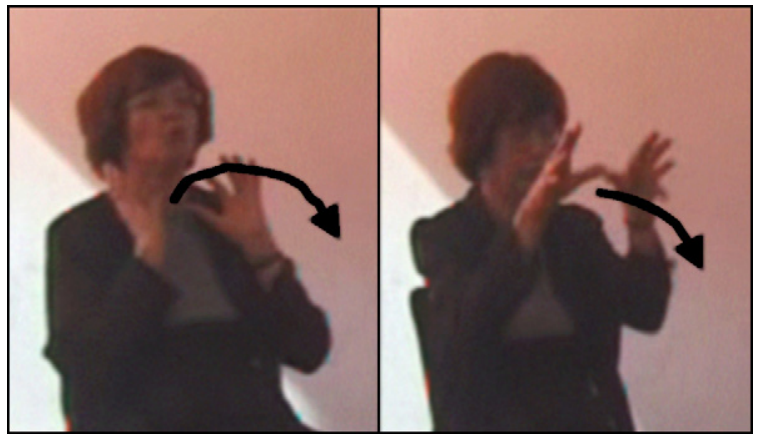

Fig. 12. Mouse(signer)-throw-ball(biman: handlingCL).

isomorphicity with the signer's body and felicitousness of representation. Thus, in her use of only prototypical character perspective signing, the signer depicts a direct figure-ground relationship only for the mouse's actual manual handling of the ball (either holding the ball, Fig. 11, or throwing the ball, Fig. 12). In these representations the ball is depicted as life-sized via a bimanual handling classifier. ${ }^{12}$

When body parts that are neither isomorphic nor felicitous for representation with the body are used to hit the ball, the signer does not use classifier forms for their representation. Instead, to represent the movement and use of these body parts (after lexically identifying them), she uses either a comparable human body part or a lexical predicate. Thus, to depict hitting the ball with the elephant's trunk and the mouse's snout, the signer moves her own head up, as though hitting the ball with her head (see Fig. 13). Though the trunk and snout are not isomorphic with her own head and nose, she uses only her own head and nose, and does not specify the shape of the trunk or

\footnotetext{
${ }^{12}$ The signer also uses a bimanual life-sized classifier form to represent the path of the ball along the sagittal axis. Though the form is the same, it is different in function from the representation of the ball being held by the mouse via a handling classifier. Technically, the path representation of the ball is a deviance from real constructed action (because the hands are representing the referent, and not the handling of the referent). However, because the size of the referent representation is life-sized, it does fall under the prototypical manifestation of character perspective signing (cf. Fig. 1, section 3.1).
} 


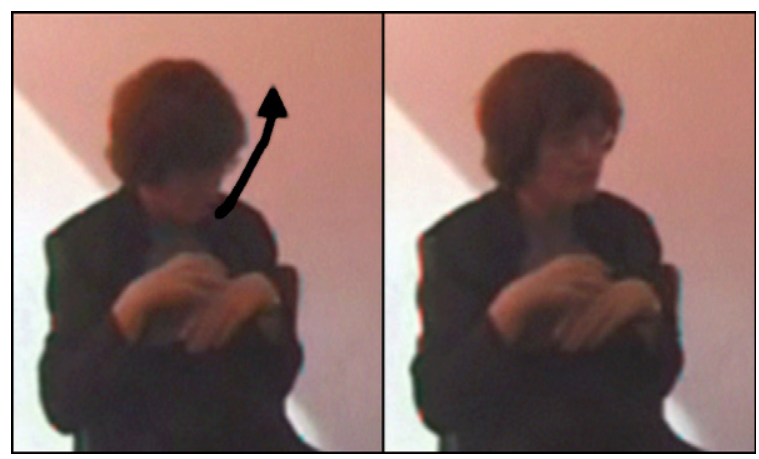

Fig. 13. Elephant(signer)-hit-ball-with-trunk(signer's head/nose).

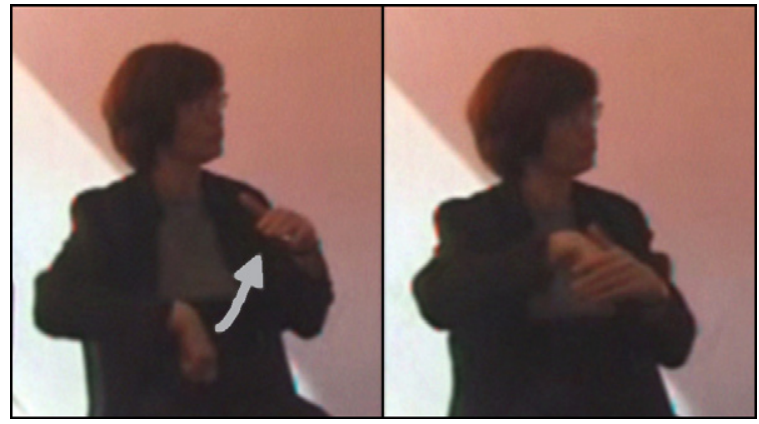

Fig. 14. Lexical predicate KICK.

the snout further using classifier forms. This is in keeping with a purely constructed action depiction of the event, where the hands represent only the hands of the animate referent mapped onto the signer's body.

When the mouse kicks the ball in the event, the relevant body part (i.e. the foot) does have an isomorphic counterpart on the human body, but it is not felicitously used in character perspective representations. ${ }^{13}$ The signer indicates the use of the foot with the lexical predicate KICK (Fig. 14). The lexical predicate is taken to occur in a separate narrative perspective. In signs ascribed to the narrator, the signer's hands are his/her own (cf. Liddell and Metzger, 1998). They do not represent referents or the hands of an animate referent, as in observer or character perspective.

Throughout the narrative, then, the signer depicts the scale of referents corresponding to a character perspective, life-sized event space. The ball is depicted exclusively with a bimanual handling form, and body parts that come into contact with the ball are represented only with isomorphic parts of the signer's own body or with a lexical predicate identifying the activity in narrator's perspective. Except for narrative signing, there is no deviation from full constructed action signing. The correspondence between perspective and classifier forms for referent representation corresponds unwaveringly to prototypical character perspective.

\footnotetext{
${ }^{13}$ The mouse in the stimulus film is personified, and thus stands upright. Its lower limbs are thus considered to be isomorphic with human feet and legs.
} 


\subsection{Depiction of scale in non-prototypical alignment}

Depending on which aspect of an event is focused upon, different classifier forms can be used to depict the same referent. The correct representation of relative scale between figure and ground objects motivates the "scale adjustment" of classifier forms. In the narrative analyzed in this section, the event space again stays life-sized, corresponding to character perspective signing. This signer, however, differs from the signer described in the previous section in that he deviates from strict constructed action, life-sized event space signing in his representation of the figure and ground referents. In particular, the signer uses limb classifiers to represent body parts that are not felicitous for representation or isomorphic with real body parts (i.e. the elephant's trunk, the mouse's snout, and the mouse's feet). Depending on the body part, the classifier form used to represent the ball (which comes into contact with the body parts) is adjusted in size. In each case, the classifier form correctly represents the relative scale between figure (i.e. the ball) and ground (i.e. the relevant body part).

For the manual handling of the ball by the mouse, the signer's representation of the ball is lifesized, because the limbs are isomorphic with his own body and can be felicitously represented in character perspective signing (Fig. 15). This does not differ from the previous signer's representation (cf. Fig. 12). For the other body parts, however, the signer uses different types of limb classifiers: extended index fingers for the legs (G-hand), curved fingers and thumb for the trunk (C-hand), and straight fingers and thumb, bent at the base joint, for the snout (flat C-hand, with an open to close movement). As observed in the quote by Slobin et al. (2003) above, the scale of the figure should correspond to the scale of the ground. Because the signer uses limb classifiers for the body parts, the life-sized body scale of prototypical constructed action signing is reduced in scale for the ground referents. Therefore, the representation of the figure referent (i.e. the ball) in each case is correspondingly reduced in scale. After the use of G-hand limb classifiers for the legs/feet kicking the ball (Fig. 16), the size of the ball is reduced in depiction to an F-hand (contact between index finger and thumb) (Fig. 17). Similarly, the C-hand representation of the elephant's trunk is matched in size by a C-hand representation of the ball (Fig. 18).

In this narrative, figure and ground referents are not represented on a life-sized scale, and thus the signer's use of classifiers manifests a non-prototypical alignment of perspective and classifier forms. Specifically, the signing here is an example of the non-prototypical classifier-perspective alignment type in which observer perspective classifiers appear in a character perspective event space (cf. Fig. 2(b), section 3.2) Through the continual scale adjustment, the narrative is highly

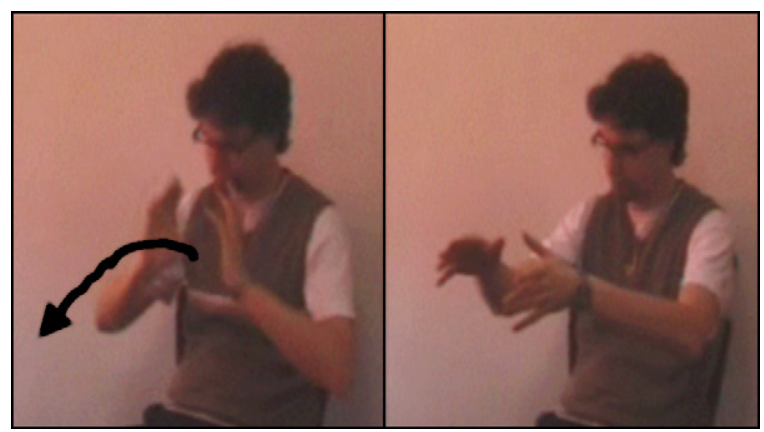

Fig. 15. Mouse(signer)-throw-ball(biman: handlingCL). 


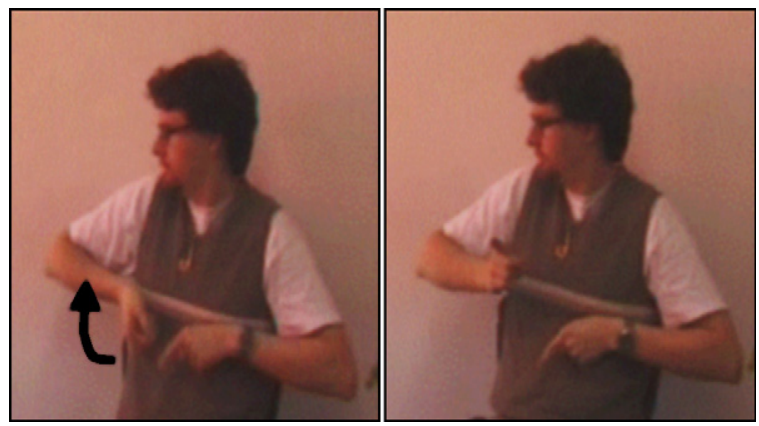

Fig. 16. Mouse(signer)-kick-ball(biman: limbCL).

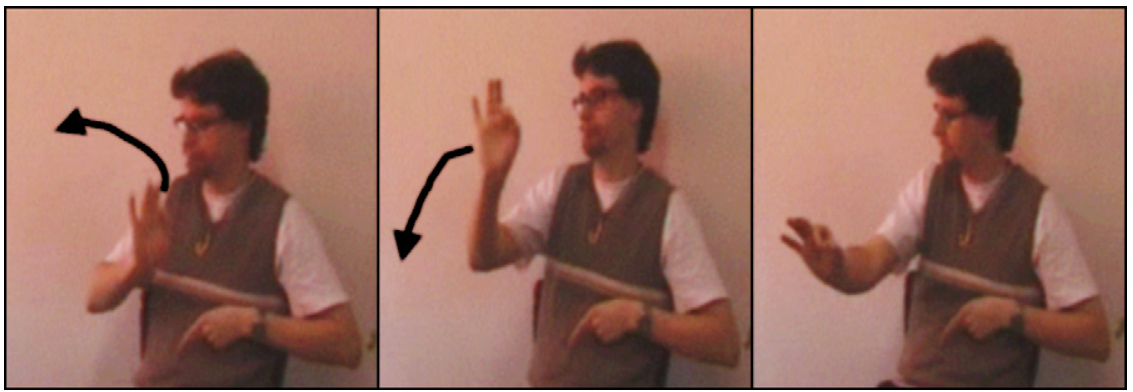

Fig. 17. Ball(RH: entityCL)-move-from-mouse(signer)-to-elephant(opp. signer).

informative with respect to the totality of spatial information (i.e. location, orientation, size, and shape).

In sum, both narratives are signed fully in character perspective, as evidenced by the mapping of animate referents on the signer's body and the use of the sagittal axis to represent the path of the ball throughout. In terms of spatial coherence, the difference between the narratives lies in the expression of locative information pertaining to size and shape. In the narrative where signing is exclusively manifested as the prototypical alignment of event space and classifier type, size and shape specification of the figure and ground referents remains minimal, because it is constrained by the size and shape of the signer's body. In contrast, the narrative that exhibits the use of

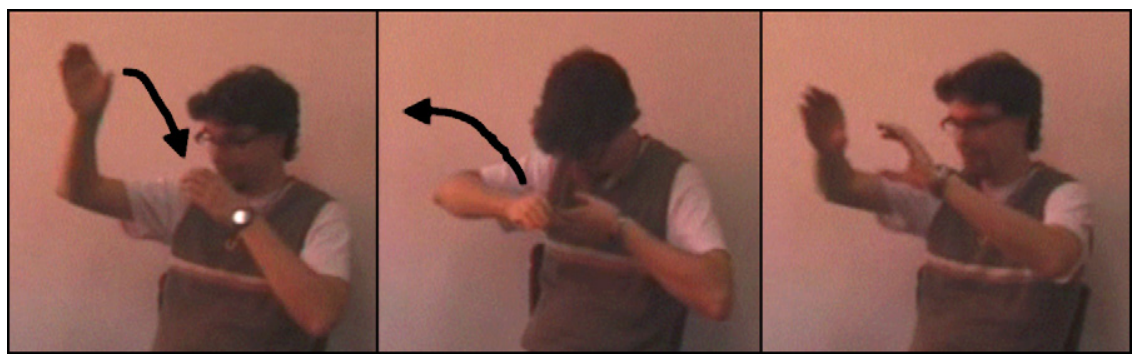

Fig. 18. Elephant(signer)-hit-ball(RH: entityCL)-with-trunk(LH: entityCL). 
classifier forms that are non-prototypically aligned with the perspective can depict information about the size and shape of referents that is much more detailed and exact. Compared to the narratives in section 5, the constancy of event space eliminates the need of mapping between different event spaces with respect to the relative locations and orientations of referents. In the expression of location and orientation information, the narratives are thus equally efficient and informative.

\section{Summary and conclusion}

In this paper, I investigated the use of classifier forms and signing perspective as spatial devices in signing that contribute crucially to spatial coherence in signed narratives. Spatial coherence was defined in terms of expressions depicting locative information about referents' locations and orientations, actions and motion, as well as features of their size and shape. Unlike most previous accounts, the use of classifier forms and perspective were treated as independent factors, which allows a unified account of their possible co-occurrences.

The differential combinations of classifier forms and perspective correspond to what I have called prototypical and non-prototypical alignments. In prototypical alignments, entity classifiers for the encoding of intransitive event types are used in an observer perspective event space projection, while handling classifiers are used to encode transitive events within an event space projected from a character perspective. In non-prototypical alignments, entity classifiers occur in a character perspective event space, handling classifiers occur in observer perspective, or both perspectives and classifier types occur simultaneously. This paper presents an analysis of the function that these forms fulfill with respect to discourse coherence. To do this, German Sign Language narratives of cartoon stimulus clips that exemplified the use of both prototypical and non-prototypical alignments were analyzed. The role of the different constructions in the achievement and maintenance of spatial coherence was illustrated. In particular, the use and function of non-prototypical alignments was elucidated with respect to the efficiency and informativeness constraints of discourse structure.

In the first analysis (section 5), I showed how the use of non-prototypical alignments contributes to discourse efficiency in the mapping of meaningful location between perspectives. Specifically, the use of prototypical classifier-perspective alignments in a default, sequentiallydetermined mapping strategy was contrasted to the more efficient, but equally informative, use of a non-prototypically aligned simultaneous perspective construction. In the second analysis (section 6), the use of non-prototypical alignments increases discourse informativeness with respect to precision in the representation of relative scale of referent size. This contrasts with the use only of prototypical constructed action: character perspective signing, where the scale of referent representation remains bound to the scale of the signer's own body. The narratives are equally efficient in their depiction of the activity engaged in by the main protagonists in the event, but the use of non-prototypically-aligned classifier-perspective constructions allows more precision in the encoding of spatial information, and is thus more informative.

Narrative discourse very frequently displays blends or combinations of elements aligned with different spaces. However, their occurrence has received little attention in the literature. Research addressing the use of non-prototypical alignments (e.g. Liddell, 2000; Dudis, 2004; Aarons and Morgan, 2003) has been primarily concerned with a formal and conceptual description of the phenomena. Here, the focus is instead on the function of non-prototypical alignments to achieve discourse coherence with respect to spatial relationships. As shown, with respect to the expression of locative information, the use of non-prototypically aligned 
constructions responds to both efficiency and informativeness constraints on discourse structure, thereby contributing to the maintenance of spatial coherence. More research remains to be done on both the form and functions of differential combinations of classifiers and perspective in extended discourse in German Sign Language, as well as other related and unrelated sign languages.

\section{Acknowledgements}

I would like to express my gratitude to the DGS signers Volker Maaßen, Anne Warnking, Isa Werth, and Uwe Zelle for their time and patience, as well as for allowing me to reproduce stills and video segments of their narratives here.

Thanks also to Aslı Özyürek and Ulrike Zeshan for help and guidance in discussing the ideas presented in this paper.

\section{Appendix A. Description of stimulus film 1 (section 5)}

The stimulus film features a personified mouse, engaged in the activity of preparing a pancake. The mouse is seen from the back, facing a stove, with its arms and shoulders moving (still 1). The mouse then turns from the stove to face left, such that the viewer can see that it is holding a pan in which it has prepared the pancake (still 2). The mouse makes numerous attempts at flipping the pancake into the air and catching it in the pan (still 3). Each time, the pancake lands on the floor instead, and each time, the mouse picks up the pancake from the floor (still 4), places it back in the pan, and turns right to face the stove again to prepare a new pancake (still 5). This sequence repeats three times.

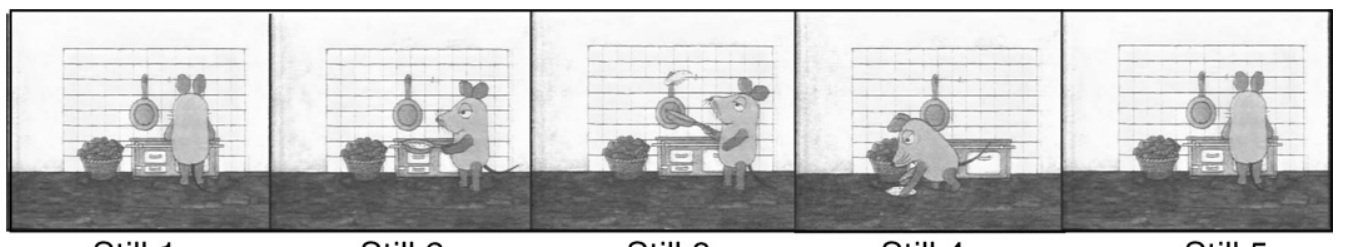

Still 1

Still 2

Still 3

Still 4

Still 5

- Still 1: Mouse faces stove (location/orientation ${ }_{1}$ ).

- Still 2: Mouse turns $90^{\circ}$ to left, holds pan with pancake in it (location/orientation 2 ).

- Still 3: Mouse moves pan upward to flip pancake into air.

- Still 4: Mouse picks pancake up from floor.

- Still 5: Mouse turns back to face stove.

\section{Appendix B. Description of stimulus film 2 (section 6)}

The stimulus film features the mouse together with a comparatively smaller elephant. The mouse and the elephant stand across from each other, playing with a ball that they pass back and forth between them. The elephant uses its trunk to hit the ball throughout the game (still 1), but 
the mouse interacts with the ball in a variety of manners. It first throws the ball (still 1), then kicks the ball, and finally, bounces the ball off of its nose.

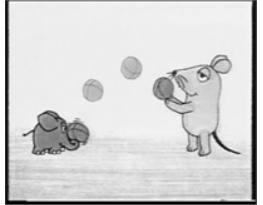

Still 1

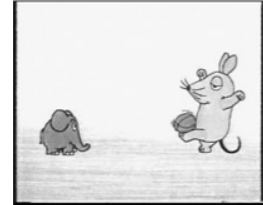

Still 2

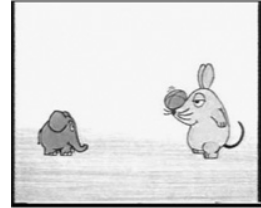

Still 3

- Still 1: Mouse throws ball, elephant hits ball with trunk.

- Still 2: Mouse kicks ball.

- Still 3: Mouse hits ball with nose.

\section{References}

Atlas, J.D., Levinson, S.C., 1981. IT-clefts, informativeness, and logical form. In: Cole, P. (Ed.), Radical Pragmatics. Academic Press, New York, pp. 1-62.

Aarons, D., Morgan, R., 2003. Classifier predicates and the creation of multiple perspectives in South African Sign Language. Sign Language Studies 3 (2), 125-156.

Bellugi, U., Klima, E., 1990. Properties of visual spatial languages. In: Prillwitz, S., Vollhaber, T. (Eds.), Sign Language Research and Application, Proceedings of the International Congress on Sign Language Research and Application, 23-25 March 1990 in Hamburg (International Studies on Sign Language and Communication of the Deaf, 13). Signum Press, Hamburg, pp. 115-144.

Benedicto, E., Brentari, D., 2000. The role of American Sign Language (ASL) verbal classifiers in argument structure. Paper presented at the Seventh International Conference on Theoretical Issues in Sign Language Research, Amsterdam, 23-27 July.

Boyes-Braem, P., 1990. Einführung in die Gebärdensprache und ihre Erforschung. Signum Press, Hamburg.

Cogill-Koez, D., 2000. Signed language classifier predicates: linguistic structures or schematic visual representation? Sign Language and Linguistics 3 (2), 153-207.

Dudis, P., 2004. Body partitioning and real-space blends. Cognitive Linguistics 15 (2), 223-238.

Emmorey, K., 1996. The confluence of space and language in signed languages. In: Bloom, P., Peterson, M.A., Nadel, L., Garrett, M.F. (Eds.), Language and Space. MIT Press, Cambridge, MA, pp. 171-209.

Emmorey, K., Falgier, B., 1998. Talking about space with space: describing environments in ASL. In: Winston, E. (Ed.), Storytelling and Conversation: Discourse in Deaf Communities. Gallaudet University Press, Washington, DC, pp. 3-26.

Engberg-Pedersen, E., 1994. Some simultaneous constructions in Danish Sign Language. In: Brennan, M., Turner, G.H. (Eds.), Word-order issues in sign language, Working Papers Presented at a Workshop Held in Durham, $18-22$ September 1991. Isla, Durham, pp. 73-87.

Engberg-Pedersen, E., 1993. Space in Danish Sign Language: The Semantics and Morphosyntax of the Use of Space in a Visual Language. Signum Press, Hamburg.

Fridman-Mintz, B., Liddell, S.K., 1998. Sequencing mental spaces in an ASL narrative. In: Koenig, J.P. (Ed.), Discourse and Cognition: Bridging the Gap. CUP, Cambridge, pp. 255-268.

Gernsbacher, M.A., 1997. Coherence cues mapping during comprehension. In: Costermans, J., Fayol, M. (Eds.), Processing Interclausal Relationships: Studies in the Production and Comprehension of Text. Erlbaum, Mahwah, NJ, pp. $3-22$.

Givón, T., 1995. Coherence in text vs. coherence in mind. In: Gernsbacher, M.A., Givón, T. (Eds.), Coherence in Spontaneous Text. Benjamins, Amsterdam, pp. 59-116.

Grice, H.P., 1975. Logic and conversation. In: Cole, P., Morgan, J.L. (Eds.), Speech Acts. Academic Press, New York, pp. 41-58. 
Horn, L.R., 1984. Toward a new taxonomy for pragmatic inference. In: Schiffrin, D. (Ed.), Meaning, Form, and Use in Context. Georgetown University Press, Washington, DC, pp. 11-42.

Levinson, S.C., 2000. Presumptive Meanings. MIT Press, Cambridge, MA.

Liddell, S.K., 1994. Tokens and surrogates. In: Ahlgren, I., Bergman, B., Brennan, M. (Eds.), Perspectives on Sign Language Structure, Papers from the fifth International Symposium on Sign Language Research, vol. 1, held in Salamanca, Spain, 25-30 May 1992. Isla, Durham, pp. 105-119.

Liddell, S.K., 1995. Real, surrogate, and token space: grammatical consequences in ASL. In: Emmorey, K., Reilly, J. (Eds.), Language, Gesture, and Space. Lawrence Erlbaum Associates, Hillsdale, NJ, pp. 19-41.

Liddell, S.K., 1998. Grounded blends, gestures, and conceptual shifts. Cognitive Linguistics 9-3, 283-314.

Liddell, S.K., 2000. Blended spaces and deixis in sign language discourse. In: McNeill, D. (Ed.), Language and Gesture: Window into Thought and Action. Cambridge University Press, Cambridge, pp. 331-357.

Liddell, S.K., 2003. Grammar, Gesture and Meaning in American Sign Language. Cambridge University Press, Cambridge.

Liddell, S.K., Metzger, M., 1998. Gesture in sign language discourse. Journal of Pragmatics 30, 657-697.

McDonald, B.H., 1982. Aspects of the American Sign Language predicate system, Unpublished doctoral dissertation. State University of New York, Buffalo.

McNeill, D., 1992. Hand and Mind: What Gestures Reveal about the Mind. University of Chicago Press, Chicago.

Meier, R.P., 1990. Person deixis in American Sign Language. In: Fischer, S.D., Siple, P. (Eds.), Theoretical Issues in Sign Language Research. University of Chicago Press, Chicago, pp. 175-190.

Metzger, M., 1995. Constructed dialogue and constructed action in American Sign Language. In: Lucas, C. (Ed.), Sociolinguistics in Deaf Communities. Gallaudet University Press, Washington, DC, pp. 255-271.

Miller, C., 1994. Simultaneous constructions in Quebec Sign Language. In: Brennan, M., Turner, G.H. (Eds.), Word-order issues in sign language, Working Papers Presented at a Workshop Held in Durham, 18-22 September 1991. Isla, Durham, pp. 89-112.

Morgan, G., 1999. Event packaging in BSL discourse. In: Winston, E. (Ed.), Storytelling and Conversation: Discourse in Deaf Communities. Gallaudet University Press, Washington, DC, pp. 27-58.

Neidle, C., 2002. SignStream: a database tool for research on visual-gestural language. Sign Language and Linguistics 4 (1/2), 203-214.

Padden, C.A., 1990. The relation between space and grammar in ASL verb morphology. In: Lucas, C. (Ed.), Sign Language Research: Theoretical Issues. Gallaudet University Press, Washington, DC, pp. 118-132.

Perniss, P.M., 2003.In: Simultaneous constructions as a device for integrating different uses of space in German Sign Language (DGS) discourse, Paper presented at the Eighth International Cognitive Linguistics Conference, Logroño, Spain, 20-25 July 2003.

Perniss, P.M., in press. Functions of simultaneity in German Sign Language narratives. In: Vermeerbergen, M., Leeson, L., Crasborn, O. (Eds.), Simultaneity in Signed Languages: Form and Function. Benjamins, Amsterdam.

Perniss, P.M., Özyürek, A., accepted. Constructing actions and locating referents: a comparison of German (DGS) and Turkish (TID) Sign Language narratives. In: Proceedings of TISLR 8, held in Barcelona, Spain, 29 September to 02 October 2004.

Schembri, A., 2003. Rethinking "classifiers" in signed languages. In: Emmorey, K. (Ed.), Perspectives on Classifier Constructions in Sign Languages. Erlbaum, Mahwah, NJ, pp. 3-34.

Schick, B., 1990. Classifier predicates in American Sign Language. International Journal of Sign Linguistics 1 (1), 1540.

Slobin, D.I., Hoiting, N., Kuntze, M., Lindert, R., Weinberg, A., Pyers, J., Anthony, M., Biederman, Y., Thumann, H., 2003. A cognitive/functional perspective on the acquisition of "Classifiers". In: Emmorey, K. (Ed.), Perspectives on Classifier Constructions in Sign Languages. Erlbaum, Mahwah, NJ, pp. 271-298.

Supalla, T., 1986. The classifier system in American Sign Language. In: Craig, C. (Ed.), Noun Classification and Categorization. John Benjamins, Philadelphia, pp. 181-213.

Supalla, T., 2003. Revisiting visual analogy in ASL classifier predicates. In: Emmorey, K. (Ed.), Perspectives on Classifier Constructions in Sign Languages. Erlbaum, Mahwah, NJ, pp. 249-258.

Sutton-Spence, R., Woll, B., 1999. The Linguistics of British Sign Language. An Introduction. CUP, Cambridge.

Talmy, L., 2003. The representation of spatial structure in spoken and signed language. In: Emmorey, K. (Ed.), Perspectives on Classifier Constructions in Sign Languages. Erlbaum, Mahwah, NJ, pp. 169-196.

Zwitserlood, I., 2003. Classifying hand configurations in Nederlandse Gebarentaal (Sign Language of The Netherlands), Doctoral dissertation. LOT, University of Utrecht, Utrecht. 